\title{
A formalization of one of the main claims of "Creative Commons licenses and the non-commercial condition: Implications for the re-use of biodiversity information" by Hagedorn et al. 2011
}

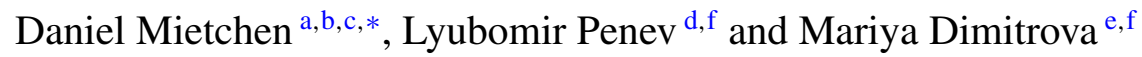 \\ ${ }^{a}$ Ronin Institute, Montclair, New Jersey, United States \\ E-mail: daniel.mietchen@ ronininstitute.org; ORCID: https://orcid.org/0000-0001-9488-1870 \\ ${ }^{\mathrm{b}}$ Leibniz Institute of Freshwater Ecology and Inland Fisheries, Berlin, Germany \\ ${ }^{\mathrm{c}}$ Freie Universität Berlin, Institute of Biology, Ecological Novelty Group, Berlin, Germany \\ ${ }^{\mathrm{d}}$ Bulgarian Academy of Sciences, Bulgaria \\ E-mail: l.penev@pensoft.net; ORCID: https://orcid.org/0000-0002-2186-5033 \\ ${ }^{\mathrm{e}}$ Bulgarian Academy of Sciences, Bulgaria \\ E-mail: m.dimitrova@pensoft.net; ORCID: https://orcid.org/0000-0002-8083-6048 \\ ${ }^{\mathrm{f}}$ Pensoft Publishers, Bulgaria
}

Editor: Cristina-Iulia Bucur (https://orcid.org/0000-0002-7114-6459)

Review comments from: Tobias Kuhn (https://orcid.org/0000-0002-1267-0234); Ricardo Usbeck

(https://orcid.org/0000-0002-0191-7211); Cristina-Iulia Bucur (https://orcid.org/0000-0002-7114-6459)

Received 7 July 2021

Accepted 3 December 2021

\begin{abstract}
Hagedorn et al. claimed in previous work that when talking about biodiversity data, licenses with a non-commercial clause generally inhibit data reuse. We present here a formalization of that claim, stating that all things of class "license with non-commercial clause" that are in the context of a thing of class "biodiversity data" generally have a relation of type "inhibits" to a thing of class "data reuse" in the same context.
\end{abstract}

Keywords: Biodiversity data, license with non-commercial clause, data reuse

\footnotetext{
${ }^{1}$ As RDF/nanopublication: http://purl.org/np/RABzhulhaPhOzo9MxWx1230N72-azdlpMNwu_HtDqsuUc

*Corresponding author. E-mail: daniel.mietchen@ ronininstitute.org.
} 


\section{Introduction}

Hagedorn et al. [2] state that "Unfortunately, in science and education, knowledge and data are often intermingled with copyrighted expressions of the same. Many publishers establish barriers to knowledge sharing by asserting copyright on non-copyrightable plain or formal expressions of that knowledge. In the area of biodiversity, often dealing with textually expressed data or data expressed in images, this is a major obstacle." We present here a formalization of the main scientific claim from this quote by using a semantic template called the super-pattern [1].

\section{Formalization}

Our formalization looks as follows:

$\begin{array}{ll}\text { CONTEXT-CLASS (“in the context of all...”): } & \text { biodiversity data } \\ \text { SUBJECT-CLASS (“things of type...”): } & \begin{array}{l}\text { license with non-commercial clause } \\ \text { Qenerally }\end{array} \\ \text { QUALIFIER: } & \text { inhibits } \\ \text { OBJECION-TYPE ("have a relation of type...”): } & \text { data reuse }\end{array}$

In the context class we use the class "biodiversity data" (Q28946370) from Wikidata. In the subject class, we use a new minted class "license with non-commercial clause" that is a subclass of "license" (Q79719) from Wikidata and is related to the class "Creative Commons NonCommercial" (Q65071627) from Wikidata. In the object class we use the class "data reuse" (Q58023280) from Wikidata.

\section{RDF code}

This is our formalization as a nanopublication in TriG format:

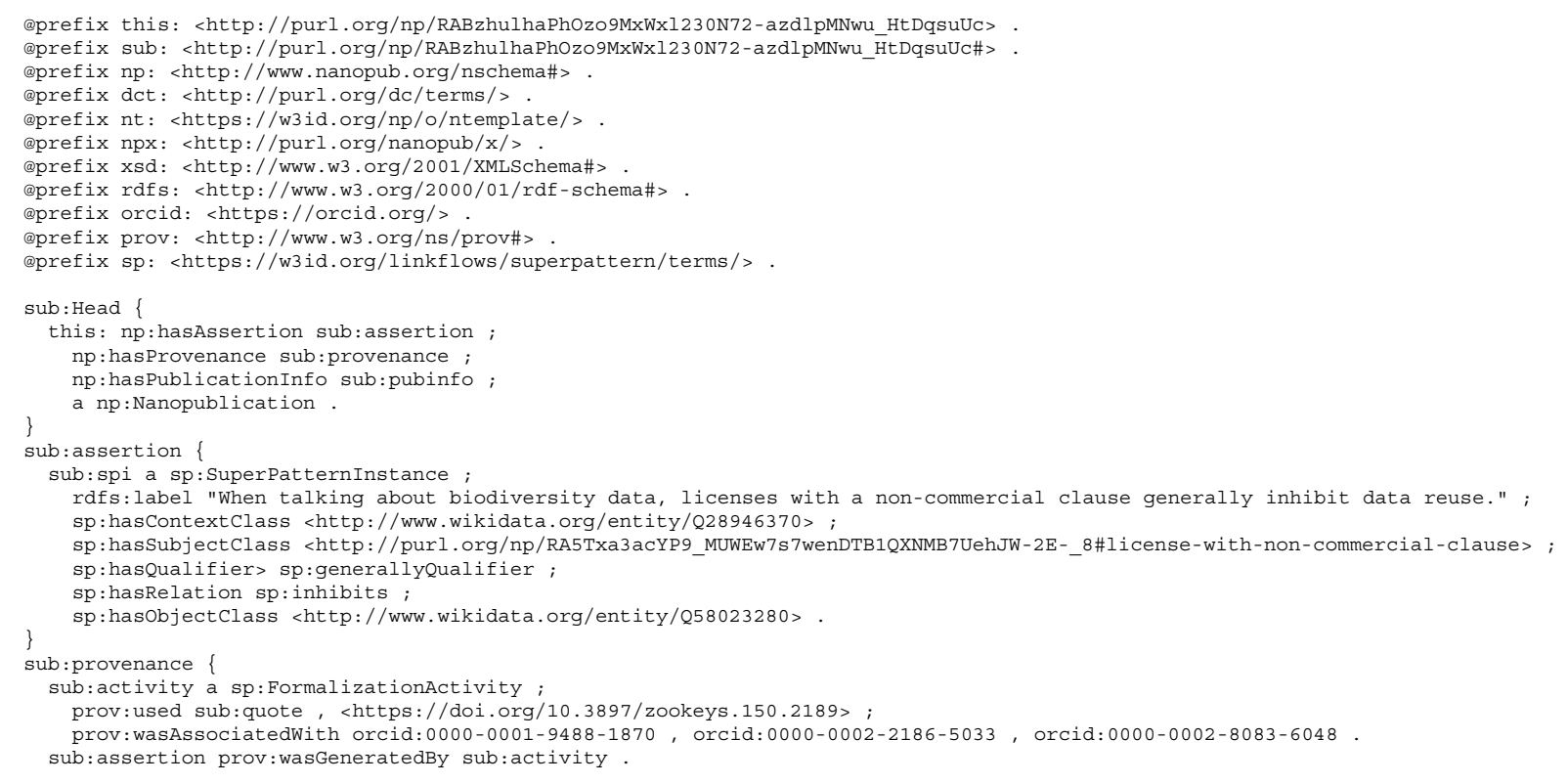


sub:quote prov:value "Unfortunately, in science and education, knowledge and data are often intermingled with copyrighted expressions of the same. Many publishers establish barriers to knowledge sharing by asserting copyright on non-copyrightable plain or formal expressions of that knowledge. In the area of biodiversity, often dealing with textually expressed data or data expressed in images, this is a major obstacle." prov: wasQuotedFrom <https://doi.org/10.3897/zookeys.150.2189>

sub: pubinfo \{

sub:sig npx:hasAlgorithm "RSA"

npx: hasPublickey

"MIGFMA0GCSGGSIb3DQEBAQUAA4 GNADCBiQKBgQCwi1YMnpV5z/0 OMXYFYDKS5cirQQWTMZ/jbglmVY1gl3YAgrkqsQUmgaXA/ho+VV8j sfREIL8PT2CzbmCjGj MJo NaKVqJq1GoIUV9+Kj 9yXxNySYDAOTDs IM0zdj1BEu5uNbt f zwQPGuhVpgLR05Uk/meCyYLPs3BxC5zRbtulmwIDAQAB"

npx: hassignature

"pqWZhyf zNdZhmUc6gaCbmq2GWJb4R/pWwk4rMv1On35Nx9y44fsz9zGWDyEEVg0frXq6EOsd5Umyq5YY1BIHFJBjiOKUaVmtxU+MuYCuTCbLp3s 1k+hY17ocAl4 0F 5SOMfCY+DqxtelrNQM5rmVQJa9wJ+mmKejz7Cs+Bm9xsD8="

npx: hassignatureTarget this:

this: dct:created "2021-11-30T21:54:51.835+02:00"^^xsd:dateTime

dct : creator orcid:0000-0002-8083-6048

npx: introduces sub:spi

npx: supersedes <http://purl.org/np/RA2hde9hXKfi0juhaF7yWdlaKsyZknPYj2Od-rLlAKmSM>

<https://w3id.org/linkflows/reviews/isUpdateOf> <http://purl.org/np/RAjk3YoNydLhbuD4BV9FD2lecELazVZibHHM7IXtJd-4A>

nt:wasCreatedFromProvenanceTemplate <http://purl.org/np/RAElwniOy0yO39PlK9QkQ-wqbC3q-R2nXraP5huu8W39k> ;

nt:wasCreatedFromPubinfoTemplate <http://purl.org/np/RA2VCBXZf-icECVRGhulJXugTGxpsV5yVr9yqCI1boh4A> , $<$ http://purl.org/np/RAA2MfqdBCzmz 9yVWj KLXNbyfBNCwsMmOqCNUxkk1maIM> ,

<http://purl.org/np/RAjpBMl w3 owYhJUBo3DtsuDlXsNAJ8cnGeWAutDVjuAuI > ;

nt:wasCreatedFromTemplate <http://purl.org/np/RAv68imZrEjfcp2rnEg1hzoBqEVc0cQMtp9_1Za0BxNM4>

\section{The following nanopublications introduce the newly minted classes in TriG format.}

This is the class definition of "license with non-commercial clause":

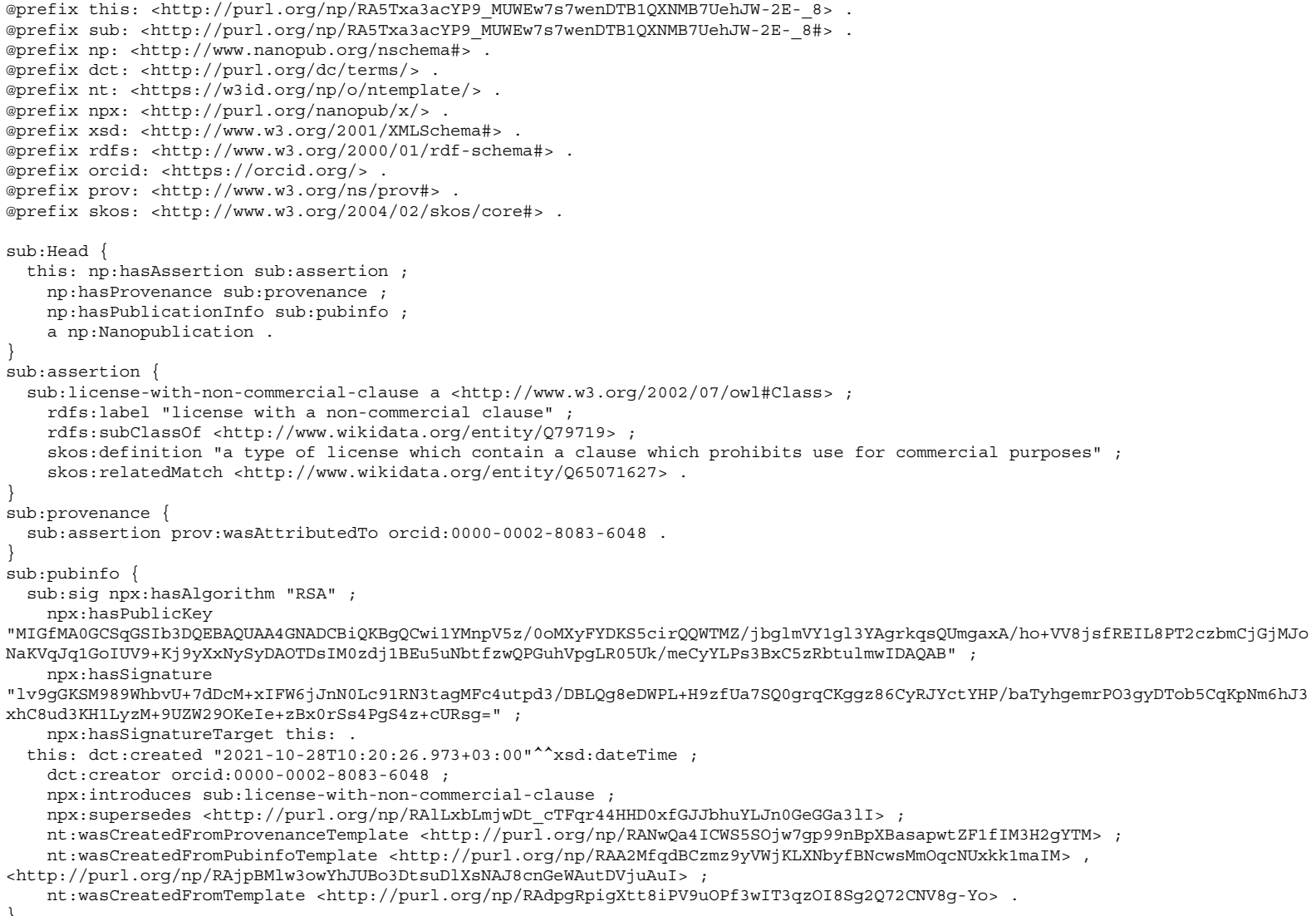




\section{References}

[1] C.I. Bucur, T. Kuhn, D. Ceolin and J. van Ossenbruggen, Expressing high-level scientific claims with formal semantics, in: Proceedings of the 11th Knowledge Capture Conference, 2021. doi:10.1145/3460210.3493561.

[2] G. Hagedorn, D. Mietchen, D. Agosti, L. Penev, W. Berendsohn and D. Hobern, Creative Commons licenses and the noncommercial condition: Implications for the re-use of biodiversity information, ZooKeys 150 (2011), 127-149. doi:10.3897/ zookeys.150.2189. 